\title{
DINAMICA COOPERATIVA IN SISTEMI COMPLESSI: CONGETTURE PER UN MODELLO DA CONFUTARE
}

\author{
MARCO PIERUCCINI (*) \\ Nota presentata dal m.e. Attilio Rigamonti \\ (Adunanza del 26 ottobre 2011)
}

SUNTO. - Viene affrontato il problema del moto molecolare in liquidi (polimerici) vicini alla temperatura di transizione vetrosa ovvero in condizioni di confinamento, quando i vincoli dinamici e strutturali ai bordi del volume occupato condizionano la dinamica interna. Per la descrizione di questa fenomenologia viene proposto un approccio termodinamico statistico che, sebbene non completo, permette di stimare grandezze come la barriera di potenziale chimico per il riarrangiamento conformazionale e le dimensioni medie delle regioni in cui tale riarrangiamento avviene, col solo ausilio di misure di rilassamento dielettrico o meccanico. Lo sviluppo delle argomentazioni è calato nel quadro filosofico proposto da Karl Popper per una visione generale della scienza e del suo rapporto con aree meno scientifiche del pensiero.

$$
* * *
$$

AвSTRACT. - The problem of the molecular motion in (polymeric) liquids approaching the glass transition or under confinement - where dynamic or structural constraints at the borders of a domain affect the bulk dynamics - is addressed. A statistical thermodynamic approach is proposed for the description of this phenomenology. Despite its incompleteness, the model allows for the estimate, from just mechanical and/or dielectric relaxation measurements, of both the chemical potential barrier for the conformational changes and the size of the regions where these changes take place. The relevant arguments are developed with constant reference to the philosophical framework proposed by Karl Popper for a general view of science and its relationship with less scientific areas of thought.

(*) CNR, Istituto Nanoscienze, v. Campi 213/A, 41125 Modena, Italy.

E-mail: marco.pieruccini@nano.cnr.it 


\section{MotivaZiOnE}

Mi sono interessato alle vedute di Karl Popper sulla teoria della conoscenza dopo diversi anni dall'inizio della mia attività. Con emozione ho ritrovato attraverso la lettura dei saggi di Popper le sensazioni e le convinzioni che mi accompagnano nel lavoro, alle quali si sono aggiunti ancor nuovi e più stimolanti spunti di riflessione.

In un contesto scientifico, fatto salvo il lato emotivo, si può considrare la mia esperienza personale come una verifica sperimentale delle idee di Popper. In senso più profondo, penso che tale esperienza ponga in rilievo la condizione umana in sé ed il pensiero che ne scaturisce, in contrapposizione alle meccaniche dell'attività scientifica che pur ne rappresentano una particolare manifestazione.

Il presente contributo vuole essere un'occasione per illustrare alcuni punti del quadro filosofico delineato da Popper attraverso la descrizione di recenti sviluppi del mio lavoro di ricerca. Certi aspetti tecnici potranno essere trattati in modo intuitivo o non rigoroso al solo scopo di rendere più facilmente comprensibile il testo ad un'ampia varietà di lettori.

\section{INTRODUZIONE}

E' opinione diffusa considerare la conoscenza scientifica il risultato di una serie di osservazioni e di deduzioni logiche. Questo modo di pensare, pur non contenendo inesattezze, potrebbe rimanere vago ed incompleto ove non si consideri che la figura del ricercatore non può confinarsi all'interno di una dimensione così impersonale, essendo la sua vocazione guidata anche dalle prerogative dello spirito, in un divenire continuo tra idee ed intuizioni la cui sintesi esprime la conoscenza.

Una visione molto più ricca, invece, scaturisce dall'analisi sviluppata da Popper [1]:

La conoscenza ha fonti di ogni genere, ma nessuna ha autorità: tutte possono condurre ad errore... Come scoprire l'errore? Criticando le teorie e i tentativi congetturali nostri e degli altri.

Vediamo allora che esistono in effetti due aspetti fondamentali della questione. Il primo è congetturale, legato in maniera più significativa a creatività e personalità, in cui la capacità di vedere analogie o di sviluppare rappresentazioni ha un ruolo dominante. E' qui che le fonti 
più diverse (teorie note, modelli anche incompleti, osservazioni sperimentali, "intuizioni") possono contribuire alla formulazione di un'idea nuova. L'altro aspetto è relativo alla critica delle congetture; qui, logica e rigore prevalgono. Le teorie più valide saranno quelle che ancora non siano state contraddette, ma non avremo mai la certezza che una teoria non lo sarà prima o poi.

La consapevolezza di questa continua contrapposizione non ci abbandona mai nell'affrontare problemi di frontiera, e ciò si verifica in particolare nel caso che è oggetto di questa nota: i moti molecolari cooperativi nei liquidi.

\subsection{La transizione vetrosa e il moto cooperativo}

Processi di raffreddamento sufficientemente rapidi determinano nei liquidi (anche polimerici), siano essi cristallizzabili o meno, una trasformazione nota come transizione vetrosa. Nello stato vetroso il sistema ha le caratteristiche macroscopiche di un solido ma, diversamente da quanto accade per un cristallo, non vi è ordine spaziale su scale di lunghezza atomiche.

Nel processo di vetrificazione, vicino alla temperatura $T_{g}$ di transizione, la resistenza opposta dal liquido allo scorrimento (la viscosità) aumenta di diversi ordini di grandezza in un intervallo di raffreddamento di pochi gradi. Poichè lo scorrimento di un liquido interessa lunghezze di correlazione $\xi$ dell'ordine dei nanometri o più (molto maggiori delle dimensioni atomiche), è naturale pensare che la transizione allo stato vetroso sia il risultato del progressivo rallentamento delle componenti (ordinate) dei moti molecolari che sono caratterizzate da tali valori di $\xi$. Se si pensa alle perturbazioni di densità associate alle fluttuazioni conformazionali, possiamo immaginare che le loro componenti di Fourier con lunghezza d'onda $l \sim \xi$ siano quasi bloccate in prossimità di $T_{g}$. In tali condizioni la conformazione molecolare media può cambiare solo in tempi enormemente lunghi (rispetto alle scale di tempo dell'esperimento), ma restano attivi gradi di libertà locali (per esempio quelli vibrazionali) che sono responsabili della capacità termica del vetro.

Se un modo dinamico con lunghezza d'onda $\xi_{0}$ è molto rallentato, allora lo sono anche quelli con $\xi>\xi_{0}$. Ad ogni temperatura $T$ esiste pertanto una regione di transizione, attorno ad un certo valore $\xi_{\text {cutoff }}(T)$, 
che separa i modi molto lenti da quelli che ancora sono attivi. Il progressivo raffreddamento verso $T_{g}$ determina una diminuzione $\operatorname{di} \xi_{\text {cutoff }}(T)$.

Lo stato di quasi congelamento delle componenti di Fourier a grande lunghezza d'onda introduce vincoli meccanici nei processi di riarrangiamento conformazionale; questi ultimi possono ancora aver luogo, ma assumono carattere cooperativo: Il movimento di una molecola (o di un'unità monomerica) è possibile solo se accade che, simultaneamente, anche quelle contenute in un suo intorno sufficientemente grande si muovano in modo opportuno; i loro spostamenti relativi sono di natura casuale e perdono il carattere collettivo tipico di un'onda, dove sono correlati in modo definito. I domini coinvolti nel riarrangiamento conformazionale, le Regioni di Riarrangiamento Cooperativo (CRR), diventano sempre più grandi quanto più ci si avvicina a $T_{g}[2]$.

\subsection{Polimeri semi-cristallini}

Diversamente da quanto avviene nei liquidi a basso peso molecolare, il processo di cristallizzazione che può aver luogo nei polimeri non è mai completo. Rimangono, confinate da cristalli, regioni amorfe costituite da tratti di catena le cui estremità spesso sono ancorate alle pareti cristalline (tratti diversi di una singola catena polimerica possono far parte di differenti domini cristallini) e si trovano generalmente in stati torsionali non compatibili con la cristallizzazione; peraltro tali tratti sono quasi totalmente aggrovigliati tra loro. Ci riferiremo in generale a queste cause come agli impedimenti topologici che non permettono che la cristallizzazione abbia luogo.

Dal punto di vista termodinamico la descrizione di una situazione di questo tipo è ancora un problema aperto. Se infatti la transizione dallo stato amorfo allo stato cristallino è accompagnata da una variazione misurabile di potenziale chimico

$$
\Delta \mu_{c r}(T)=\mu_{c}-\mu_{a}<0,
$$

dove $\mu_{c}$ è il potenziale nello stato cristallino e $\mu_{a}$ è quello nella fase amorfa sottoraffreddata, è pur vero che non si conosce il modo di misurare il potenziale chimico nelle regioni amorfe confinate, né il criterio termodinamico che soggiace all'arresto del processo di cristallizzazione.

Poichè il potenziale chimico $\mu_{a}$ è tanto più basso quanto più alta è l'entropia per unità monomerica (cioè, il sistema è tanto più stabile 
termodinamicamente quanto maggiore è il numero di stati che esso può esplorare per un dato valore della sua energia), è chiaro che l'effetto dei vincoli topologici e del confinamento in generale contribuirà ad un aumento del potenziale chimico della fase amorfa rispetto al valore $\mu_{a 0}$ in assenza totale di vincoli:

$$
\overline{\Delta \mu} \equiv \mu_{a}-\mu_{a 0}>0 .
$$

$E^{\prime}$ a questo punto che una prima congettura prende forma: L'arresto del processo di cristallizzazione è probabile sia regolato da una sorta di equilibrio tra la forza $\Delta \mu_{c r}$ responsabile della transizione e $\overline{\Delta \mu}$, che è associato alla presenza di vincoli conformazionali (topologici). Se così è, allora le caratteristiche della dinamica conformazionale devono contenere informazione dello stato termodinamico dei domini confinanti, cioè i cristalli.

Vi è un altro aspetto di notevole interesse nei sistemi polimerici semicristallini: Nelle regioni amorfe confinate - diciamo di dimensioni lineari tipiche $a$ - non possono svilupparsi fluttuazioni conformazionali ordinate con lunghezza d'onda $l \gtrsim a$, essendo i cristalli all'intorno relativamente rigidi. Esiste allora una lunghezza tipica $\xi_{\text {cutoff }} \sim a$ che separa i modi attivi da quelli bloccati. La situazione presenta una forte analogia con quella esposta nella sezione precedente, ed è per questo che ci si aspetta che anche in queste condizioni il moto molecolare abbia carattere cooperativo. Ma non è solo questa seconda congettura a rendere interessante il problema: l'induzione controllata della cooperatività (in questo senso i cristalli rappresenterebbero di fatto una sorta di strumento di misura) potrebbe permettere di comprendere più a fondo il meccanismo della transizione vetrosa.

\section{UN MODELLO PER LA STATISTICA DELLE CRR}

La formulazione di un modello per un sistema fisico richiede uno sforzo di astrazione; è questo che crea i presupposti per poter immaginare collegamenti con altre idee e stabilire analogie con altri sistemi. L'interpretare un fenomeno consiste di fatto nel ricondurlo ad altri già noti, magari apparentemente scorrelati.

Immaginiamo un liquido in cui le fluttuazioni conformazionali, che sono dovute allo scambio di energia con l'ambiente, hanno carattere 
cooperativo. Non interessa per ora da cosa sia indotta la cooperatività (tanto più che non abbiamo ancora verificato se il confinamento ne sia veramente una possibile causa), consideriamo però il sistema come una popolazione di CRR nelle quali i riarrangiamenti conformazionali avvengono a tempi casuali. Supporremo, per semplicità, che le CRR consistano tutte dello stesso numero $z$ di monomeri (trascuriamo quindi la dispersione attorno a $z$, salvo poi tenerne eventualmente conto in uno sviluppo successivo del modello).

Per definizione, una transizione conformazionale cooperativa rimane circoscritta ad una CRR e non determina direttamente variazioni conformazionali al di fuori di essa. I moti locali veloci invece, come quelli vibrazionali, interagiscono sia coi modi conformazionali che con il bagno termico, senza essere "schermati" in alcun modo. In altre parole, $i$ gradi di libertà conformazionali sono in contatto termico con l'ambiente (e perciò sono supporto di fluttuazioni) solo attraverso i gradi di libertà locali.

Consideriamo ora una CRR in un determinato stato conformazionale metastabile, cioé in uno stato $A$ caratterizzato da determinati vincoli topologici, che richiede l'attraversamento di una barriera energetica $z \zeta_{A}$ per transire ad un nuovo stato (metastabile) $B$. La fluttuazione di energia necessaria al superamento della barriera è garantita dal contatto termico con l'ambiente attraverso i gradi di libertà locali più veloci. Una volta raggiunto il nuovo stato conformazionale, e quindi una diversa condizione vincolare, la fluttuazione minima di energia $z \zeta_{B}$ necessaria per uscirne sarà in generale diversa dalla prima, i.e. $\zeta_{B} \neq \zeta_{A}$. La Fig. 1 riproduce schematicamente l'andamento in funzione del tempo dell'energia libera di una CRR che transisce da uno stato conformazionale all'altro. Di fatto $\zeta$ parametrizza i vincoli conformazionali di una CRR, essendo l'energia minima (per monomero) necessaria per abbandonare una determinata condizione vincolare. Quest'ultimo aspetto è rilevante perché stabilisce un'associazione tra i vincoli topologici dentro una CRR e l'energia minima di barriera $\zeta$, che può essere naturalmente inquadrata in ambito termodinamico.

La Fig. 2 illustra in forma diversa l'idea di una CRR i cui vincoli conformazionali (e quindi $\zeta$ ) fluttuano nel tempo: lo stato 1 può essere abbandonato solo attraverso una transizione ad uno stato intermedio di mobilità, ad esempio lo stato 2; successivamente la CRR può transire 


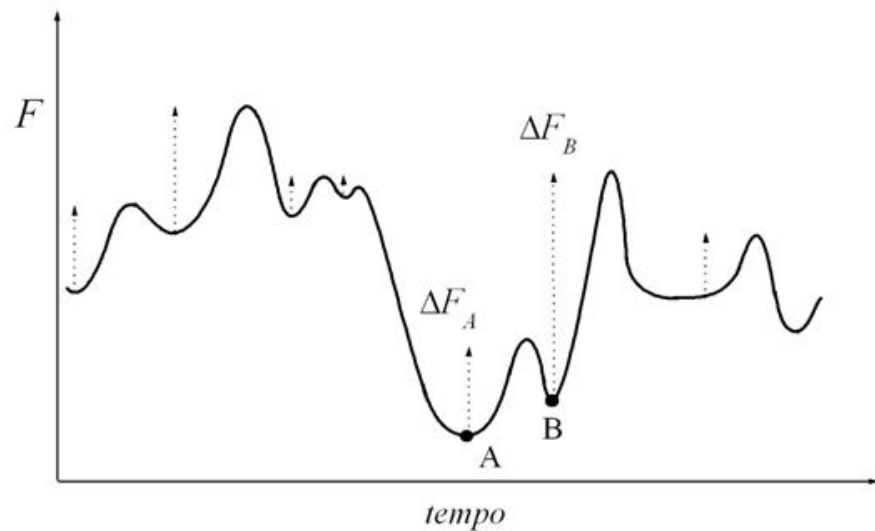

Fig. 1 - Fluttuazioni dell'energia libera $F$ di una CRR nel corso del tempo. Ciascun minimo relativo corrisponde in principio ad un diverso stato conformazionale che è possibile abbandonare se ba luogo una fluttuazione di energia superiore o uguale ad un valore minimo di soglia dipendente anch'esso dallo stato della CRR.

ad un altro stato tra molti possibili. Di lì il processo si ripete. (Paragonando le Fig. 1 e 2 bisogna tener conto che in generale $z \zeta \neq \Delta F$, come apparirà chiaro dalla forma della probabilità di transizione $q(\zeta)$ definita in seguito.)

La dinamica cooperativa dell'intero sistema è quindi rappresentata come un insieme di CRR le cui conformazioni fluttuano incessantemente ed in modo mutuamente scorrelato; il parametro $\zeta$ associato ad ognuna di esse (che si ipotizza possa assumere qualsiasi valore non negativo) fluttua di conseguenza. In uno stato stazionario l'insieme di CRR sarà quindi distribuito nel dominio della variabile $\zeta$ secondo una densità $p(\zeta)$ invariabile nel tempo; il numero di CRR con parametro di vincolo nell'intervallo $d \zeta$ attorno al valor medio $\zeta$ sarà proporzionale a $p(\zeta) d \zeta$.

Un aspetto importante da sottolineare nella rappresentazione che conduce alla Fig. 2 è il fatto che non vi sia conoscenza del valore di $\zeta$ che caratterizzerà la CRR una volta lasciato lo stato 2 di mobilità (questo vale a partire da un qualunque stato di mobilità). In altre parole, una volta abbandonato lo stato di mobilità, qualsiasi valore di $\zeta$ nel nuovo stato metastabile è possibile. La traduzione di questa indeterminatezza nel linguaggio formale passa attraverso la definizione dell'entropia di mis- 


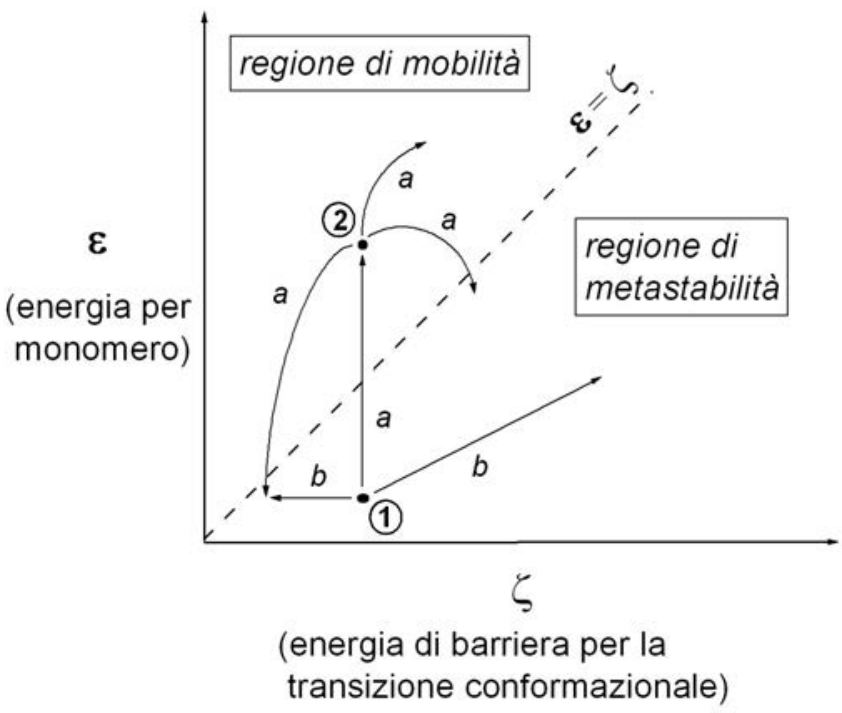

Fig. 2 - Regioni di mobilità e di metastabilità di una CRR. $\varepsilon \equiv E / z$ è l'energia per monomero. Affinché una transizione possa avvenire è necessario che la CRR acquisisca (per fluttuazione) un'energia che la porti nello stato di mobilità (transizione $1 \rightarrow 2$ ); transizioni contrassegnate con "b" non sono possibili.

cela associata alla varietà di stati (cioè di $\zeta$ ) che una CRR può assumere:

$$
S_{\zeta}[p]=-k_{B} \int_{0}^{+\infty} d \zeta p(\zeta) \ln [p(\zeta)],
$$

dove $k_{B}$ è la costante di Boltzmann. Seguendo le linee indicate dalla meccanica statisica, la condizione di massima indeterminazione corrisponde alla distribuzione $p(\zeta)$ per cui l'entropia $S_{\zeta}[p]$ è massima, sotto i vincoli che l'energia media $U \equiv U[p]$ negli stati di mobilità ed il potenziale chimico $\overline{\Delta \mu}$ siano costanti.

Tralasciando i dettagli dello sviluppo, per i quali si rimanda al riferimento bibliografico [3], riportiamo qui il risultato cui si perviene:

$$
p(\zeta) \sim e^{-\frac{\bar{\varepsilon} \zeta}{k_{B} T}-\lambda \Delta \mu(\zeta)}
$$


dove $\bar{\varepsilon}_{\zeta}$ è l'energia media (per monomero) sugli stati di mobilità per un fissato valore di $\zeta$,

$$
\Delta \mu(\zeta) \equiv-\frac{k_{B} T}{z} \ln \left[\frac{\sum_{E \geq z \zeta} e^{-E / k_{B} T}}{\sum_{E} e^{-E / k_{B} T}}\right]
$$

(con $E$ l'energia totale di fluttuazione della CRR) è il potenziale chimico nello stato di mobilità associato alla barriera di energia $z \zeta$, e $\lambda$ è il moltiplicatore di Lagrange legato alla condizione $\overline{\Delta \mu}=$ cost. imposta per il calcolo dell'estremo di $S_{\zeta}[p]$ sul potenziale chimico medio

$$
\overline{\Delta \mu}=\int_{0}^{\infty} d \zeta p(\zeta) \Delta \mu(\zeta)
$$

L'aspetto più interessante dell'espressione di $p(\zeta)$ ottenuta è la forma poissoniana rispetto al potenziale chimico $\Delta \mu(\zeta)$. Questo ci dice che la dispersione $\delta[\Delta \mu]$ di $\Delta \mu(\zeta)$ attorno al valor medio $\overline{\Delta \mu}$ è dell'ordine del valor medio stesso (non è proprio uguale per effetto del fattore di Boltzmann $\left.e^{-\bar{\varepsilon}_{\zeta} / k_{B} T}\right)$ :

$$
\delta[\Delta \mu] \approx \overline{\Delta \mu} .
$$

Questa relazione pone in luce due importanti aspetti:

- moltiplicando ambo i membri per $z$ otteniamo a destra dell'equazione l'energia libera media $\Delta F=z \overline{\Delta \mu}$ associata alle fluttuazioni conformazionali di una CRR, mentre a sinistra abbiamol'ampiezza della dispersione media di questa stessa grandezza. Il fatto che il valor medio di una grandezza estensiva (in questo caso $F$ ) sia paragonabile all'ampiezza delle sue fluttuazioni è indicativo di un sistema termodinamicamente piccolo;

- la misura dello stato termodinamico $\overline{\Delta \mu}$ delle regioni amorfe confinate è possibile, essendo ricondotta alla misura di $\delta[\Delta \mu]$ che, come si vedrà, è accessibile in modo relativamente semplice attraverso misure di rilassamento dielettrico o meccanico.

Fino a questo punto l'equazione 7 non è altro che una congettura. Essa eredita questo attributo dall'ipotesi che $\zeta$ possa assumere qualsiasi 
valore a partire da uno stato qualunque di mobilità. Ovviamente non è questa l'unica ragione; nel determinare $p(\zeta)$ abbiamo per esempio fatto ricorso ad un'analogia tra l'insieme delle CRR (caratterizzate ognuna da uno stato $\zeta$ e da un'energia media $\bar{\varepsilon}_{\zeta}$ nello stato di mobilità) ed una collezione di sistemi identici non mutuamente interagenti nell'ambito del formalismo canonico [4].

\section{Misura DEL POTENZIALE $\overline{\Delta \mu}$}

Misure di risposta meccanica o dielettrica in frequenza eseguite in condizioni isoterme possono fornire una stima per il potenziale $\overline{\Delta \mu}$ ed il numero di unità monomeriche $z$ che formano le CRR. Questo verrà illustrato considerando il ruolo della funzione di rilassamento temporale delle fluttuazioni locali di polarizzazione o di conformazione.

\subsection{Spettroscopia dielettrica}

L'applicazione di un campo elettrico oscillante (sufficientemente debole) $\mathrm{E}(t)=\mathrm{E}_{0} e^{i \omega t}$ induce in un sistema (polimerico) una polarizzazione $\mathrm{P}(t)=\mathrm{P}_{0} e^{i \omega t-i \varphi}$, dove $\varphi$ è un possibile ritardo di fase. Queste grandezze sono legate linearmente tra loro dalla relazione

$$
\mathrm{P}(t)=\epsilon_{0}\left[\epsilon^{*}(\omega)-1\right] \mathrm{E}(t),
$$

in cui $\epsilon_{0}$ è la costante dielettrica del vuoto ed $\epsilon^{*}(\omega) \equiv \epsilon^{\prime}(\omega)-i \epsilon^{\prime \prime}(\omega)$ è la costante dielettrica relativa, la cui dipendenza dalla frequenza $\omega$ del campo è legata ai diversi processi molecolari che hanno luogo nel sistema. Se tali processi sono indipendenti, nello spirito di un approccio puramente fenomenologico si può esprimere $\epsilon^{*}(\omega)$ come somma di diversi contributi:

$$
\epsilon^{*}=\sum_{n} \epsilon_{n}^{*} \sim \sum_{n} \frac{\Delta \epsilon_{n}}{\left[1+\left(i \omega \tau_{n}\right)^{a_{n}}\right]^{b_{n}}},
$$

dove $\Delta \epsilon_{n}$ è l'ampiezza del contributo $n$-simo, $\tau_{n}$ è il suo tempo caratteristico, $a_{n}(>0)$ è l'esponente di allargamento in frequenza e $\left.\left.b_{n} \in\right] 0,1\right]$ è il parametro di asimmetria. 
Da ognuno di tali contributi si può ricavare la funzione di rilassamento associata

$$
\phi_{n}^{D}(t)=\frac{2}{\pi} \int_{0}^{\infty} \frac{\epsilon_{n}^{\prime \prime}(\omega)}{\Delta \epsilon_{n}} \cos (\omega t) \frac{d \omega}{\omega},
$$

cioè la funzione che descrive come il sistema perde memoria del suo stato iniziale (per esempio una fluttuazione locale della polarizzazione) per effetto di quel preciso meccanismo molecolare [5].

Siamo qui interessati in particolare agli effetti dovuti alla riorientazione di dipoli permanenti associata a transizioni conformazionali.

\subsection{Spettroscopia meccanica}

Un discorso analogo si può fare nei confronti della spettroscopia meccanica. Consideriamo per fissare le idee un sistema polimerico sottoposto ad uno sforzo di tensione $\sigma(t)=\sigma_{0} e^{i \omega t}$. La risposta meccanica sarà una deformazione $d(t)=d_{0} e^{i \omega t-i \varphi}$, ed il ruolo di $\epsilon^{*}(\omega)$ nella spettroscopia dielettrica è qui ricoperto dal rapporto

$$
D^{*}(\omega) \equiv D^{\prime}-i D^{\prime \prime} \equiv \frac{d(t)}{\sigma(t)} .
$$

Anche qui, in presenza di moti molecolari (quasi) indipendenti, si potrà esprimere $D^{*}(\omega)$ in termini di somme di contributi in forma analoga all'equazione 9 e la funzione di correlazione $\phi_{n}^{M}(t)$ associata al processo $n$-simo si otterrà sostituendo $D^{\prime \prime}(\omega)$ al posto di $\epsilon^{\prime \prime}(\omega)$ nell'equazione 10.

\subsection{Funzione di correlazione conformazionale per un insieme di CRR}

Consideriamo una CRR in un dato stato metastabile $\zeta$; l'abbandono di questo stato è un evento casuale cui è associata la probabilità di transizione per unità di tempo

$$
q(\zeta)=\nu^{*} e^{-\frac{z \Delta \mu(\zeta)}{k_{B} T}}
$$

dove $\nu^{*}$ è la frequenza caratteristica con la quale i gradi di libertà che scambiano energia direttamente col bagno termico stimolano una transizione conformazionale (si noti, dal confronto con l'equazione 5 , che il 
termine esponenziale non è altro che la somma delle probabilità che la CRR abbia energia maggiore della soglia minima $z \zeta$ ). Un insieme di CRR identiche perderà quindi memoria dello stato iniziale secondo la legge di decadimento esponenziale $\sim e^{-q(\zeta) t}$, dove $t$ è il tempo. Quest'ultima è di fatto la funzione di correlazione (temporale) associata al processo di transizione da uno stato definito $(\zeta)$ a tutti gli altri possibili. La generalizzazione immediata per un insieme di CRR distribuite secondo $p(\zeta)$ porta ad una funzione di correlazione della forma

$$
\phi(t)=\int_{0}^{\infty} d \zeta p(\zeta) e^{-q(\zeta) t} .
$$

Il caso di una distribuzione $p(\zeta)=\delta\left(\zeta-\zeta_{0}\right)$, che porta al decadimento esponenziale considerato poco sopra, si otterrebbe dalla trasformata di Fourier (cfr. eq. 10) di una funzione analoga alla 9, ma composta da un solo termine con $a=b=1$.

\subsection{Analisi dei dati}

La Fig. 3 illustra la scomposizione di uno spettro dielettrico ottenuto da polietilene tereftalato (PET), cristallizzato alla temperatura $T_{c}=100{ }^{\circ} \mathrm{C}$ per 7 ore a partire dallo stato vetroso $\left(T_{g} \simeq 76^{\circ} \mathrm{C}\right)$. Iniziando dalle frequenze più basse dello spettro si osservano due processi simmetrici di rilassamento $(b=1)$ centrati attorno ad $1 \mathrm{~Hz}$ e $100 \mathrm{~Hz}$ (processi $\alpha$ ). Essi sono associati a riorientazioni di dipoli collegate a transizioni conformazionali delle catene polimeriche nelle regioni amorfe. $\mathrm{Si}$ ritiene $[6,7]$ che il processo a frequenza media più bassa, $\alpha_{s}$, abbia luogo in zone amorfe in cui i vincoli conformazionali sono relativamente più efficaci. La simmetria dei processi di rilassamento è da collegarsi al meccanismo fisico di confinamento da parte dei cristalli [8]. All'estremità destra dello spettro è visibile la coda di un processo veloce (processo $\beta$ ), che è associato a fluttuazioni orientazionali dipolari a livello locale non collegate a riarrangiamenti conformazionali di catena.

La Fig. 4 mostra tre spettri meccanici ottenuti alle temperature di 95, 90 e $85^{\circ} \mathrm{C}$ da PET cristallizzato a $100^{\circ} \mathrm{C}$. Anche in questo caso ogni traccia consiste principalmente di due contributi, $\alpha_{w}$ ed $\alpha_{s}$, analoghi a quelli osservati nella risposta dielettrica. Si noti come al diminuire della 


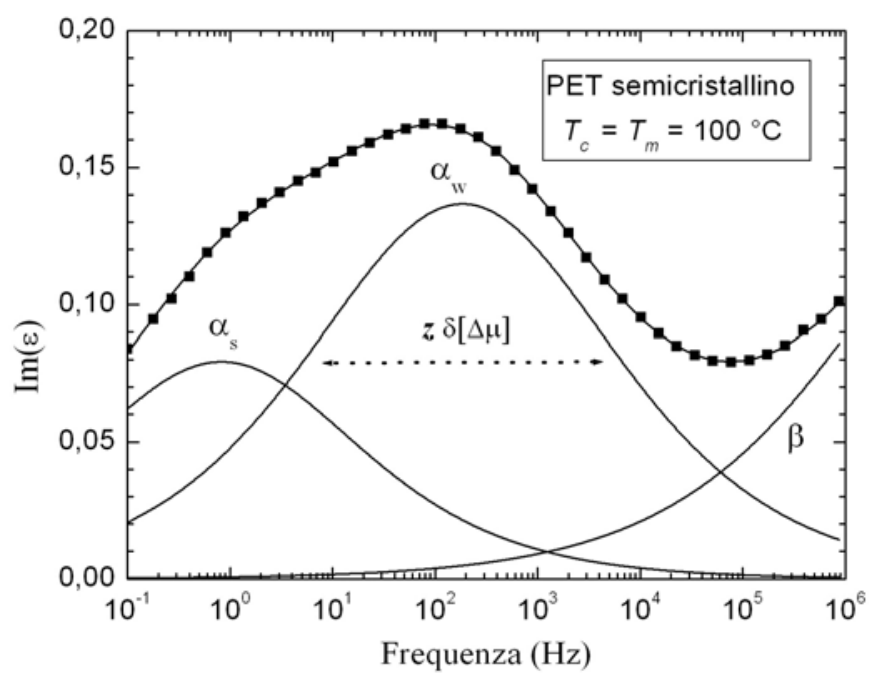

Fig. 3 - Parte immaginaria della costante dielettrica relativa in funzione della frequenza per un campione di polietilene tereftalato semicristallino. La temperatura di misura $\grave{e} T_{m}=T_{c}=100{ }^{\circ} \mathrm{C}$.

temperatura il profilo della risposta trasli verso le frequenze più basse, mentre allo stesso tempo l'allargamento in frequenza delle componenti $\alpha_{w}$ aumenti (le componenti $\alpha_{s}$ non si possono isolare in modo altrettanto ben definito per $T=85$ e $90^{\circ} \mathrm{C}$, perciò non verranno considerate nel seguito); poiché l'allargamento in frequenza è legato a $z \delta[\Delta \mu]$, per l'equazione 7 questo andamento è indicativo di un aumento della barriera di energia libera di riarrangiamento al diminuire della temperatura.

Una volta definite le diverse componenti dello spettro (dielettrico o meccanico), è immediato ricavare le relative funzioni di correlazione attraverso l'equazione 10. Da ognuna di esse è possibile allora estrarre informazioni su $z$ e $\overline{\Delta \mu}$ adattandovi la funzione di rilassamento modello dedotta sopra. Un'ipotesi essenziale che facciamo è quindi

$$
\phi(t) \sim \phi^{M}(t) \sim \phi^{D}(t) .
$$




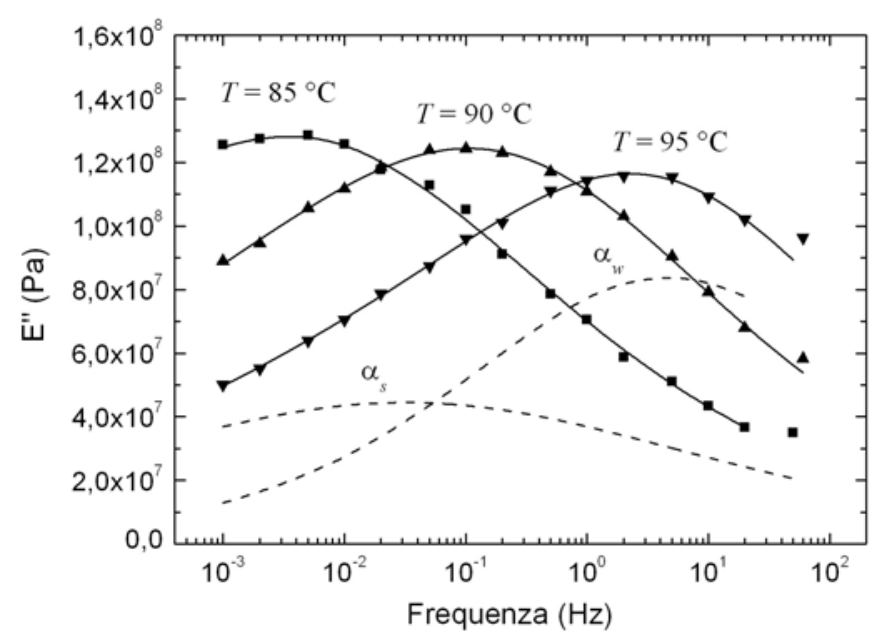

Fig. 4 - Parte immaginaria della risposta meccanica in funzione della frequenza per il campione di polietilene tereftalato semicristallino di cui alla Fig. 3. Le temperature di misura sono $T_{m}=95,90 \mathrm{ed} 85^{\circ} \mathrm{C}$. Lo spettro ottenuto a $95^{\circ} \mathrm{C}$ è stato scomposto nei due processi simmetrici $\alpha_{w}$ ed $\alpha_{s}$ analogamente a quanto fatto per lo spettro dielettrico.

La Fig. 5 mostra come la funzione $\phi(t)$ si adatta alle funzioni di correlazione associate ad $\alpha_{w}$ ed $\alpha_{s}$ nel caso di rilassamento dielettrico (Fig. 3). I dati più rilevanti, estratti con questa procedura, sono riportati nella tabella 1 . La tabella 2 contiene invece i risultati ottenuti dagli spettri meccanici di Fig. 4. In tutti i casi considerati, la dimensione media delle CRR rimane nell'ordine di $1-2 \mathrm{~nm}$.

\section{DiSCUSSIONE DEI RISULTATI}

Vale la pena ricordare i nodi principali del discorso sviluppato fin qui. Da una parte è stata fatta l'ipotesi che il confinamento di regioni amorfe da parte di cristalli induca una dinamica di tipo cooperativo. Dall'altra si è sviluppato (per la sua verifica) un modello che consente di estrarre informazioni importanti come il potenziale $\overline{\Delta \mu}$ e la dimensione delle CRR $(z)$ dall'analisi di dati. Poichè il modello stesso è fondato su ipotesi suscettibili di verifica (che portano ad esempio alla equazione 4, a sua volta utilizzata nell'equazione 13 per ottenere i risultati riportati 


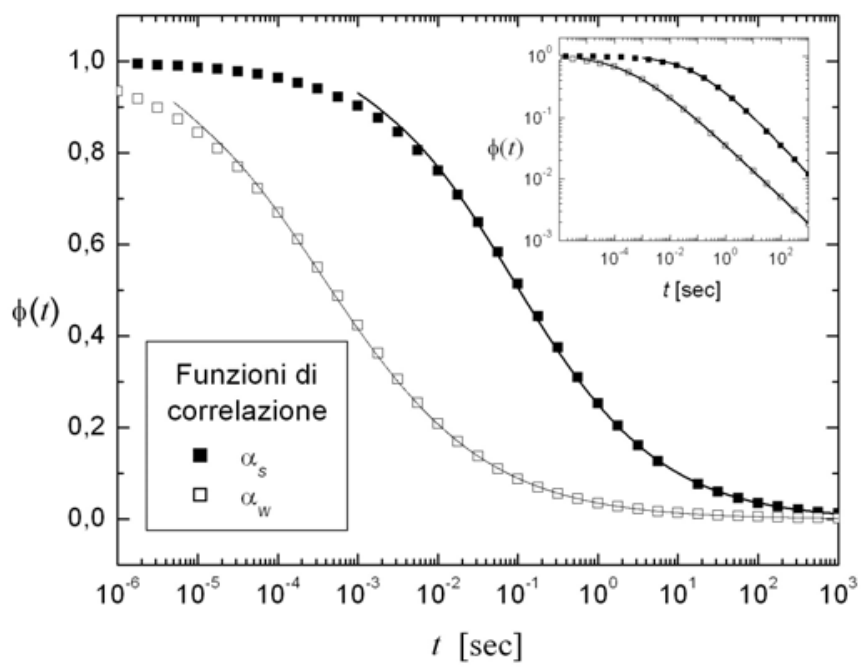

Fig. 5 - Funzioni di correlazione $\phi_{n}^{D}(t)$ estratte dai dati di Fig. 3 (simboli) e migliori adattamenti con la funzione definita dall'equazione 13 (linee continue). Nel riquadro interno le stesse funzioni sono rappresentate in scala bilogaritmica.

nelle tabelle), siamo davanti ad una situazione non del tutto lineare, ma senz'altro più frequente di quanto non si creda. Ciò in cui possiamo sperare è che le nostre ipotesi nel loro complesso (inclusa quindi l'analisi precedente) portino a risultati non contraddittori.

Il carattere cooperativo della dinamica conformazionale si manifesta attraverso l'aumento di $z$ e dell'energia libera di riarrangiamento $\Delta F=z \overline{\Delta \mu}$ al diminuire della temperatura. In particolare, la viscosità $\eta$ dipende dall'energia di riarrangiamento secondo la relazione $\eta \sim$ $\left[\nu^{*}(T)\right]^{-1} e^{\Delta F / k_{B} T}$ (cf. anche l'equazione 12). Come accennato nell'introduzione, queste caratteristiche sono proprie di un liquido prossimo a $T_{g}$ e sono senz' altro verificate dai dati riportati nelle tabelle 1 e 2 che, ricordiamo, sono stati ottenuti in base al modello sviluppato più sopra (è importante sottolineare che negli intervalli di temperatura considerati la morfologia delle strutture semicristalline resta invariata). 
Tab. 1 - Tempi di rilassamento medio $\tau_{\alpha}$ eparametri di allargamento a per $i$ processi $\alpha_{w}$ e $\alpha_{s}$ (cfr. Fig. 3) ad una temperatura di $100{ }^{\circ} \mathrm{C}$. Parametri come il moltiplicatore di Lagrange $\lambda$, il numero di monomeri $z$ in una $C R R$, così come $\overline{\Delta \mu}, \delta[\Delta \mu]$ e $\Delta F \equiv z \overline{\Delta \mu}$ si ottengono dalla procedura di adattamento di $\phi(t)$ a $\phi^{D}(t)$.

\begin{tabular}{|c|c|c|c|c|c|c|c|c|}
\hline & $\begin{array}{l}\tau_{\alpha} \\
\text { (s) }\end{array}$ & $a$ & $\begin{array}{l}\lambda \\
(\mathrm{kcal} / \\
\mathrm{mol})^{-1}\end{array}$ & $\begin{array}{l}{\left[\nu^{*}\right]^{-1}} \\
(\mathrm{~s})\end{array}$ & $z$ & $\begin{array}{l}\overline{\Delta \mu} \\
\mathrm{kcal} / \\
\mathrm{mol}\end{array}$ & $\begin{array}{l}\delta[\Delta \mu] \\
\mathrm{kcal} / \\
\mathrm{mol}\end{array}$ & $\begin{array}{l}\Delta F \\
\mathrm{kcal} / \\
\mathrm{mol}\end{array}$ \\
\hline $\begin{array}{l}\text { compo- } \\
\text { nente } \\
\alpha_{w}\end{array}$ & $\begin{array}{l}8.6 \times \\
10^{-4}\end{array}$ & 0.41 & 1.54 & $\begin{array}{l}3.6 \times \\
10^{-6}\end{array}$ & 2.7 & 1.56 & 0.96 & 4.2 \\
\hline $\begin{array}{l}\text { compo- } \\
\text { nente } \\
\alpha_{s}\end{array}$ & 0.19 & 0.45 & 1.77 & $\begin{array}{l}8.1 \times \\
10^{-4}\end{array}$ & 2.7 & 1.55 & 0.88 & 4.1 \\
\hline
\end{tabular}

I dati raccolti in questo contributo non consentono di porre direttamente in risalto come una dinamica cooperativa possa essere indotta dal confinamento da parte dei cristalli $\mathrm{o}$, più specificamente, dalla presenza di vincoli conformazionali dovuti all'ancoraggio delle catene alla frontiera dei domini amorfi. Per far questo è necessario per esempio considerare il rilassamento in sistemi confinati in nanopori. Non è possibile esporre questo studio nei dettagli, ma qualche informazione può essere trovata nel riferimento bibliografico [7] relativamente al polidimetil siloxano. Il risultato fondamentale è che al diminuire delle dimensioni dei nanopori (dell'ordine di $5 \div 20 \mathrm{~nm}$ ) l'ampiezza in frequenza della componente $\alpha$ del rilassamento dielettrico aumenta sensibilmente. Alla luce dell'equazione 7 sappiamo che questo indica un aumento della barriera di energia libera $\Delta F$ per il riarrangiamento, osservandosi un ben definito aumento anche in $z$.

Un supporto indiretto all'ipotesi che il confinamento da parte dei cristalli induce cooperatività si può trovare nel confronto tra i valori ottenuti per $\overline{\Delta \mu}$ e la variazione di potenziale chimico $\Delta \mu_{c r}$ su campioni cristallizzati isotermicamente fino ad arresto completo del processo. Ad 
Tab. 2 - Tempi di rilassamento medio $\tau_{\alpha}$ e parametri di allargamento a per $i$ processi $\alpha_{w}$ e $\alpha_{s}$ a diverse temperature $T$ per un campione di PET cristallizzato a $T_{c}=100{ }^{\circ} \mathrm{C}$ per 7 ore. Dalle procedure di adattamento di $\phi(t)$ a $\phi^{M}(t)$ si ottengono $i$ valori di $\nu^{*}, z, \overline{\Delta \mu}, \delta[\Delta \mu]$ e $\Delta F \equiv z \overline{\Delta \mu}$ riportati.

\begin{tabular}{|c|c|c|c|c|c|c|c|c|}
\hline & $\begin{array}{l}\mathrm{T} \\
\left({ }^{\circ} \mathrm{C}\right)\end{array}$ & $\begin{array}{l}\tau_{f} \\
(\mathrm{sec})\end{array}$ & $a$ & $\begin{array}{l}{\left[\nu^{*}\right]^{-1}} \\
(\mathrm{sec})\end{array}$ & $z$ & $\begin{array}{l}\overline{\Delta \mu} \\
\mathrm{kcal} / \\
\mathrm{mol}\end{array}$ & $\begin{array}{l}\delta[\Delta \mu] \\
\mathrm{kcal} / \\
\mathrm{mol}\end{array}$ & $\begin{array}{l}\Delta F \\
\mathrm{kcal} / \\
\mathrm{mol}\end{array}$ \\
\hline \multirow[t]{3}{*}{$\begin{array}{l}\text { compo- } \\
\text { nente } \\
\alpha_{w}\end{array}$} & 95 & $\begin{array}{l}3.5 \times \\
10^{-2}\end{array}$ & 0.37 & $\begin{array}{l}5.3 \times \\
10^{-4}\end{array}$ & 3.6 & 1.34 & 0.82 & 4.86 \\
\hline & 90 & 0.64 & 0.34 & $\begin{array}{l}3.4 \times \\
10^{-4}\end{array}$ & 5 & 1.09 & 0.64 & 5.4 \\
\hline & 85 & 50 & 0.31 & $\begin{array}{l}2.5 \times \\
10^{-3}\end{array}$ & 11.3 & 0.57 & 0.31 & 6.43 \\
\hline $\begin{array}{l}\text { compo } \\
\text { nente } \\
\alpha_{s}\end{array}$ & 95 & $\begin{array}{l}5.6 \times \\
10^{-2}\end{array}$ & 0.35 & $\begin{array}{l}5.3 \times \\
10^{-5}\end{array}$ & 12.4 & 0.62 & 0.36 & 7.67 \\
\hline
\end{tabular}

un valore finito di $\overline{\Delta \mu}$ è infatti collegata la cooperatività, ed una correlazione tra $\overline{\Delta \mu}$ e $\Delta \mu_{c r}$ suggerirebbe la presenza dei domini cristallini come origine di tale cooperatività.

La variazione di potenziale chimico per cristallizzazione è data approssimativamente da [9]

$$
\Delta \mu_{c r}(T) \simeq H_{f 0} \frac{T-T_{m}}{T_{m}} \frac{T}{T_{m}},
$$

dove $H_{f 0}$ è l'entalpia di fusione di un monocristallo e $T_{m}$ è la temperatura di fusione all'equilibrio. Nel caso del PET alla temperatura $T=T_{c}=100{ }^{\circ} \mathrm{C}$ si ottiene $\left|\Delta \mu_{c r}(T)\right| \simeq 1.4 \mathrm{kcal} / \mathrm{mol}$, che è in ottimo accordo coi valori ottenuti per $\overline{\Delta \mu}$ sia per il processo $\alpha_{w}$ che per l' $\alpha_{s}$ (cfr. tabella 1). Va notato che lo stesso valore di $\overline{\Delta \mu}$ si ottiene per tutt'e 
due i processi rilassamentali; ciò indica che dal punto di vista conformazionale l'effetto del confinamento da parte dei cristalli è lo stesso e la sola differenza tra i due sta in $\nu^{*}$ (l'origine di questa differenza, così come altri aspetti della teoria che per brevità non sono stati considerati, è tutt'ora un problema aperto).

Lo stesso tipo di verifica è stato fatto su un certo numero di polimeri semirigidi cristallizzati isotermicamente fino al termine del processo e successivamente analizzati dal punto di vista dielettrico ad una temperatura molto vicina a quella di cristallizzazione. La Fig. 6 mostra la correlazione tra $\overline{\Delta \mu} \mathrm{e}\left|\Delta \mu_{c r}\right|$ (cfr. il riferimento bibliografico [8] per maggiori dettagli). Questi risultati sostengono fortemente, almeno per i sistemi considerati, non solo che il confinamento induce cooperatività nella dinamica conformazionale, ma anche che un possibile criterio termodinamico per l'arresto del processo di cristallizzazione è

$$
\overline{\Delta \mu} \approx\left|\Delta \mu_{c r}\right| .
$$

Naturalmente tutto ciò può soltanto rappresentare un suggerimento per sviluppi futuri: servirà quanto meno da punto di partenza per l'interpretazione di ogni anomalia che si dia la possibilità di osservare. In una prospettiva più prossima, invece, queste osservazioni motivano uno sforzo per costruire una teoria che connetta la transizione verso una dinamica cooperativa allo spegnimento di modi normali (collettivi) a grande lunghezza d'onda.

\section{CONSIDERAZIONI FINALI}

Le ipotesi sono il contenuto essenziale di questo contributo; lo è quella dell'induzione della cooperatività per confinamento, così come quelle alla base del modello meccanico-statistico per l'interpretazione dei dati. Questi ultimi sostengono l'ipotesi sul confinamento, che però resta una congettura perchè ricondotta alla consistenza del modello.

Questo processo non ha fine, ed in linea generale esclude la possibilità di conoscenze certe. Qual è allora il senso di ciò che noi consideriamo conoscenza? A mio modo di vedere, l'essenza della nostra idea di conoscenza è l'insieme coerente di schemi mentali (teorie... ipotetiche naturalmente, e più o meno formalizzate) e di osservazioni 


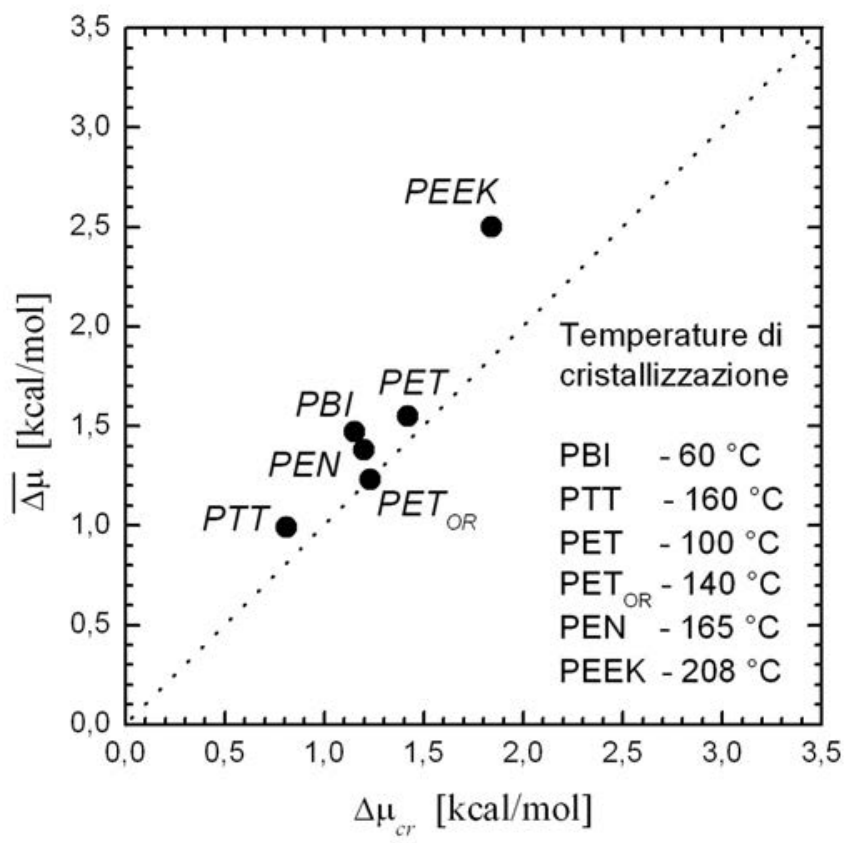

Fig. 6 - Correlazione tra $\overline{\Delta \mu}$ e $\left|\Delta \mu_{c r}\right|$ per: polietilene tereftalato non orientato (PET), polietilene tereftalato orientato a freddo prima della cristallizzazione (PETOR), polietilene naftalato (PEN), polibutilene isoftalato (PBI), poli eter eter chetone (PEEK) e politrimetilene tereftalato (PTT).

sperimentali. Ecco che allora la costante verifica delle teorie o, a dirla con Popper [1], i tentativi di confutare le nostre congetture (attraverso osservazioni ad esempio), rappresentano un passo essenziale per l'ampliamento della conoscenza, che si concretizza poi con lo sviluppo di teorie coerenti con un insieme più vasto di osservazioni.

La spiegazione di un fenomeno sulla base di teorie note può essere il risultato di un percorso difficile, ma senz'altro appagante, non foss'altro che per essere riusciti a comprendere in un'unica visione osservazioni apparentemente scorrelate (notiamo ad esempio che nel formulare il modello per la dinamica cooperativa su base termodinamicostatistica, implicitamente chiamiamo a sostegno delle nostre congetture 
tutte le osservazioni che corroborano la meccanica statistica in sé). Un attimo di riflessione però ci porta a pensare che in realtà non si è scoperto nulla di veramente nuovo e paradossalmente abbiamo la sensazione che il progresso del pensiero scientifico, così come il pensiero scientifico in sé, si fondi sul dubbio. La contrapposizione tra apparenza e realtà (ove mai esista), così vividamente messa in luce dalla meccanica quantistica ad esempio, sembra quindi essere ubiqua e non altro che una manifestazione di una condizione più profonda, insita nella natura umana.

\section{RICONOSCIMENTI}

Ho avuto la fortuna di conoscere il Professor Attilio Rigamonti quando ero studente all'Università di Pavia; egli è stato inconsapevole riferimento per me in tutti questi anni. Vorrei ringraziarlo per avermi dato l'opportunità di esprimermi attraverso questo contributo. Ringrazio anche mia moglie Adriana per le ampie discussioni relative agli aspetti filosofici di questa nota e per i consigli di carattere stilistico all'atto della sua redazione. 


\section{RIFERIMENTI BIBILIOGRAFICI}

[1] K.R. Popper (2009) Congetture e confutazioni; Il Mulino.

[2] G. Adam, J.H. Gibbs, On the Temperature Dependence of Cooperative Relaxation Properties in Glass-Forming Liquids, J. Chem. Phys. 43 (1965) 139-146.

[3] M. Pieruccini, T.A. Ezquerra, Segmental relaxation in semicrystalline polymers: A mean-field model for the distribution of relaxation times in confined regimes, Eur. Phys. J. E 29 (2009) 163-171.

[4] W. Greiner, L. Neise, H. Stocker (1995) Thermodynamics and Statistical Mechanics; Springer-Verlag.

[5] G. Williams, Time-correlation Functions and Molecular Motion, Chem. Soc. Rev. 7 (1978) 89-131

[6] C. Alvarez, I. Šics, A. Nogales, Z. Denchev, S.S. Funari, T.A. Ezquerra, Structuredynamics relationship in crystallizing poly(ethylene terephthalate) as revealed by time-resolved X-ray and dielectric methods, Polymer 45 (2004) 3953-3959.

[7] P. Calandra, S. Sturniolo, M. Pieruccini, The emergence of cooperative dynamics in polymers as an effect of conformational restrictions: The case of crystallization and an example on heterogeneous confinement, Therm. Acta 522 (2011) 135-143

[8] M. Pieruccini, A. Flores, The influence of crystal formation on segmental mobility in polymers: hints from a statistical mechanical relaxation model, Coll. Polym. Sci. 288 (2010) 365-376.

[9] J.D. Hoffman, Thermodynamic driving force in nucleation and growth processes, J. Chem. Phys. 29 (1958) 1192-1193 